\title{
Hepcidin-mediated iron sequestration protects against bacterial dissemination during pneumonia
}

\author{
Kathryn R. Michels, ${ }^{1}$ Zhimin Zhang, ${ }^{2}$ Alexandra M. Bettina, ${ }^{1}$ R. Elaine Cagnina, ${ }^{2}$ Debora Stefanova, ${ }^{3}$ \\ Marie D. Burdick, ${ }^{2}$ Sophie Vaulont, ${ }^{4}$ Elizabeta Nemeth, ${ }^{5}$ Tomas Ganz, ${ }^{5}$ and Borna Mehrad ${ }^{1,2,6}$ \\ 'Departments of Microbiology, Immunology, and Cancer Biology, ${ }^{2}$ Division of Pulmonary \& Critical Care Medicine, \\ Department of Medicine, University of Virginia, Charlottesville, Virginia, USA. ${ }^{3}$ Departments of Molecular, Cellular, and \\ Integrative Physiology, University of California, Los Angeles, California, USA. ${ }^{4}$ INSERM U1016, Cochin Institute, Descartes \\ University, Paris, France. ${ }^{5}$ Department of Medicine, David Geffen School of Medicine, University of California, Los Angeles, \\ California, USA. ${ }^{6}$ Beirne B. Carter Center for Immunology, University of Virginia, Charlottesville, Virginia, USA.
}

\begin{abstract}
Gram-negative pneumonia is a dangerous illness, and bacterial dissemination to the bloodstream during the infection is strongly associated with death. Antibiotic resistance among the causative pathogens has resulted in diminishing treatment options against this infection. Hepcidin is the master regulator of extracellular iron availability in vertebrates, but its role in the context of host defense is undefined. We hypothesized that hepcidin-mediated depletion of extracellular iron during Gram-negative pneumonia protects the host by limiting dissemination of bacteria to the bloodstream. During experimental pneumonia, hepcidin was induced in the liver in an IL-6dependent manner and mediated a rapid decline in plasma iron. In contrast, hepcidin-deficient mice developed a paradoxical increase in plasma iron during infection associated with profound susceptibility to bacteremia. Incubation of bacteria with iron-supplemented plasma enhanced bacterial growth in vitro, and systemic administration of iron to WT mice similarly promoted increased susceptibility to bloodstream infection. Finally, treatment with a hepcidin analogue restored hypoferremia in hepcidin-deficient hosts, mediated bacterial control, and improved outcomes. These data show hepcidin induction during pneumonia to be essential to preventing bacterial dissemination by limiting extracellular iron availability. Hepcidin agonists may represent an effective therapy for Gram-negative infections in patients with impaired hepcidin production or signaling.
\end{abstract}

Conflict of interest: EN and TG are inventors of minihepcidins, hold a patent on these peptides (US20120040894 A1), and are scientific founders and shareholders in Merganser Biotech, a company involved in the commercial development of minihepcidins.

Submitted: December 19, 2016 Accepted: February 9, 2017 Published: March 23, 2017

\section{Reference information:} JCI Insight. 2017;2(6):e92002. https:// doi.org/10.1172/jii.insight.92002.

\section{Introduction}

Infections caused by aerobic Gram-negative bacteria are prevalent and dangerous. As a group, these organisms are the most common etiology of nosocomial infections, including most cases of hospital-acquired pneumonia, ventilator-associated pneumonia and healthcare-associated pneumonia, and they are important causes of morbidity and death in hospitalized patients (1). Dissemination of these organisms from the primary site of infection to the bloodstream is especially associated with mortality $(2,3)$. The progressive rise in antibiotic resistance among Gram-negative bacteria, particularly in the healthcare setting, has critically diminished the available treatment options for these infections. Better mechanistic understanding of the host-pathogen interplay during Gram-negative infections has the potential to lead to novel therapeutic strategies to augment the diminishing armamentarium of traditional antibiotics.

Iron is an essential component of many proteins and a required nutrient for nearly all organisms. In biological systems, free iron ions are present at extremely low concentrations, and the competition for iron between pathogens and the host is important to the outcome of many infections (4). While the iron acquisition mechanisms of aerobic Gram-negative bacteria are known to be essential to their virulence (5), the host mechanisms of iron restriction in these infections are incompletely defined.

Under homeostatic conditions, plasma iron is rapidly turned over with a half-life of $<1$ hour, being continuously utilized in erythropoiesis and replenished by splenic macrophages that scavenge iron from senescent erythrocytes (6). The hormone hepcidin is the key regulator of plasma iron concentration that acts by mediating the degradation of the only known cellular iron exporter, ferroportin, thus trapping 
A

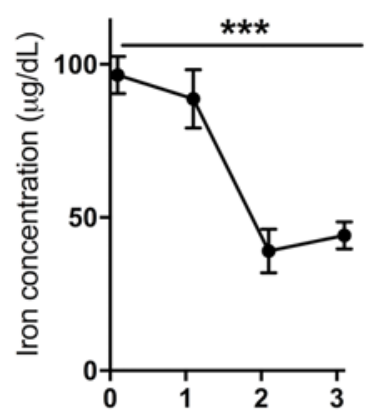

D Iron treatment

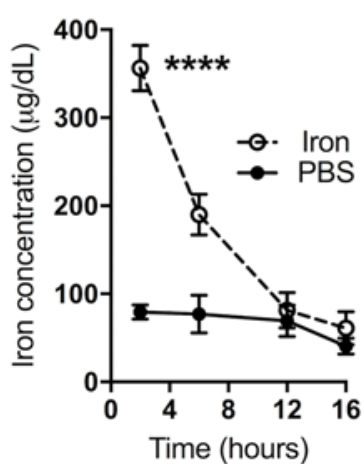

B

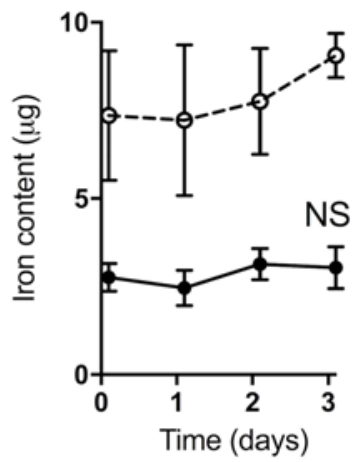

E

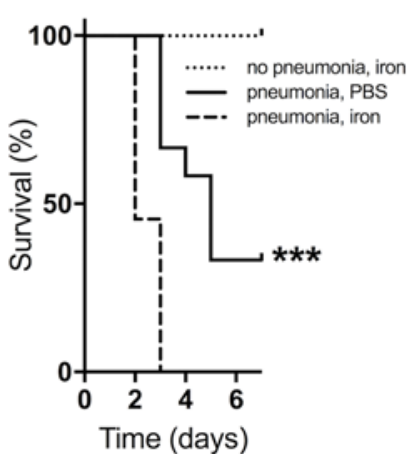

C $\quad B A L$
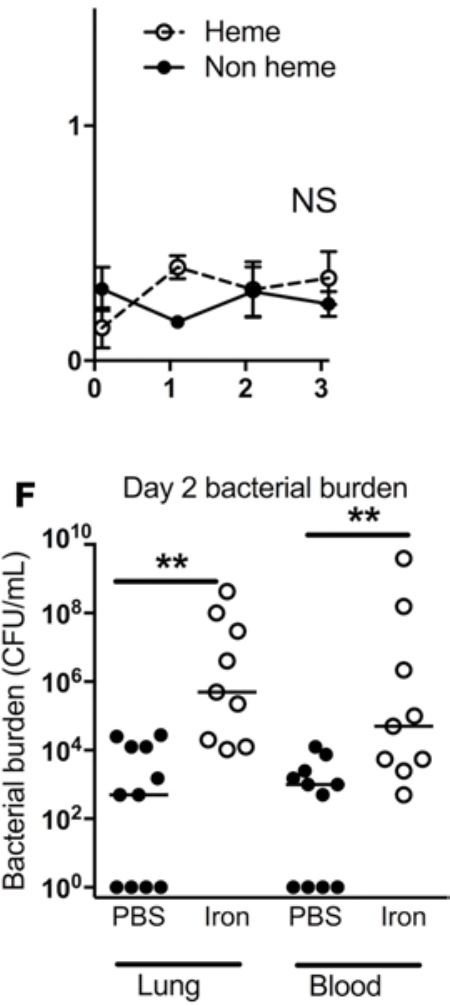

Figure 1. Iron availability during Klebsiella pneumonia. Time 0 represents uninfected animals. (A) Iron concentration in the plasma. (B and $\mathbf{C}$ ) Calculated total iron levels in whole lung homogenate per mouse, and bronchoalveolar lavage fluid (BAL) recovered in a volume of $1 \mathrm{ml}$ per mouse. Data shown as mean $\pm \mathrm{SE} ; n=4-6$ animals per time point, representative of 2 independent experiments; ${ }^{* *} P<0.001$ (1-way ANOVA, multiple comparison for linear trend). (D) Plasma iron following i.p. injection with PBS or ferric ammonium citrate (iron) 30 minutes prior to intrapulmonary $K$. pneumoniae challenge. $n=6-8$ per group per time point, combined result of 2 experiments; ${ }^{* * *} P<0.0001$, two-way ANOVA. (E) Mice were treated with either PBS or ferric ammonium citrate (iron) 30 minutes prior to intrapulmonary K. pneumoniae challenge and again 24 hours thereafter. $n=6-12$ per group, combined result of 2 experiments; ${ }^{* *} P<0.001$ Mantel-Cox test. (F) Bacterial burden on day 2 of infection in mice treated with ferric ammonium citrate or PBS prior to intratracheal challenge with $K$. pneumonia. Horizontal lines represent median, and each circle represents 1 animal; animals with no detectable bacteria are reported to have a bacterial burden of 1 CFU on the logarithmic scale. $n=9-11$, combined result of 2 experiments; ${ }^{* *} P<0.01$ Mann-Whitney $U$ test.

iron inside cells and reducing extracellular iron (7). During homeostasis, hepcidin is induced by elevated extracellular and intracellular iron levels via SMAD4 signaling, and is inhibited by anemia and hypoxia. During inflammation, hepcidin is induced as an acute-phase reactant by IL- 6 and Activin B via STAT3 and SMAD4, respectively $(8,9)$. In the context of inflammation, hepcidin mediates acute hypoferremia, which is posited to be a defense mechanism against infectious disease; continual hepcidin production in this setting can infamously cause anemia of chronic inflammation. The role of hepcidin in disorders of iron metabolism is well established, but its contribution to antimicrobial host defenses is an emerging field. For example, hepcidin appears to protect the host against malaria but to promote the growth of pathogens that reside within the intracellular niche of macrophages (10). Hepcidin-mediated hypoferremia is essential for protection of the host against Vibrio vulnificus in a murine model (11), consistent with the clinical observation that individuals with iron-overload conditions are uniquely susceptible to this organism (12). In contrast, the role of hepcidin as a general defense against common bacterial infections, such as those caused by Enterbacteriaceae, remains unknown.

We reasoned that the bloodstream dissemination of microorganisms from the primary site of infection is a critical complication in Gram-negative bacterial infections. In the context of pneumonia, as well as other focal infections caused by aerobic Gram-negative bacilli, bacteremia is strongly associated with sepsis and death $(13,14)$. We therefore tested the hypothesis that hepcidin-mediated depletion of extracellular iron during Gram-negative pneumonia protects the host by limiting dissemination of bacteria to the bloodstream.

\section{Results}

Plasma iron is suppressed during bacterial pneumonia. We used a well-described model of Klebsiella pneumonia caused by a virulent strain of bacteria, delivered directly into the trachea of sedated experimental mice ( 15 , 16). We first characterized the effect of Klebsiella pneumonia on iron metabolism in WT mice. As expected, plasma iron concentration decreased quickly in WT mice following infection (Figure 1A), similar to prior reports with acute inflammatory stimuli (9). To assess the availability of iron to bacteria in the lungs, we also measured heme-associated and non-heme-associated iron levels in whole lung homogenates (which includes both intra- and extracellular iron), and in the extracellular space, in the bronchoalveolar lavage (BAL) supernatant (Figure 1, B and C). We found that most lung iron was intracellular, and extracellular iron levels were low and did not change appreciably during infection. 

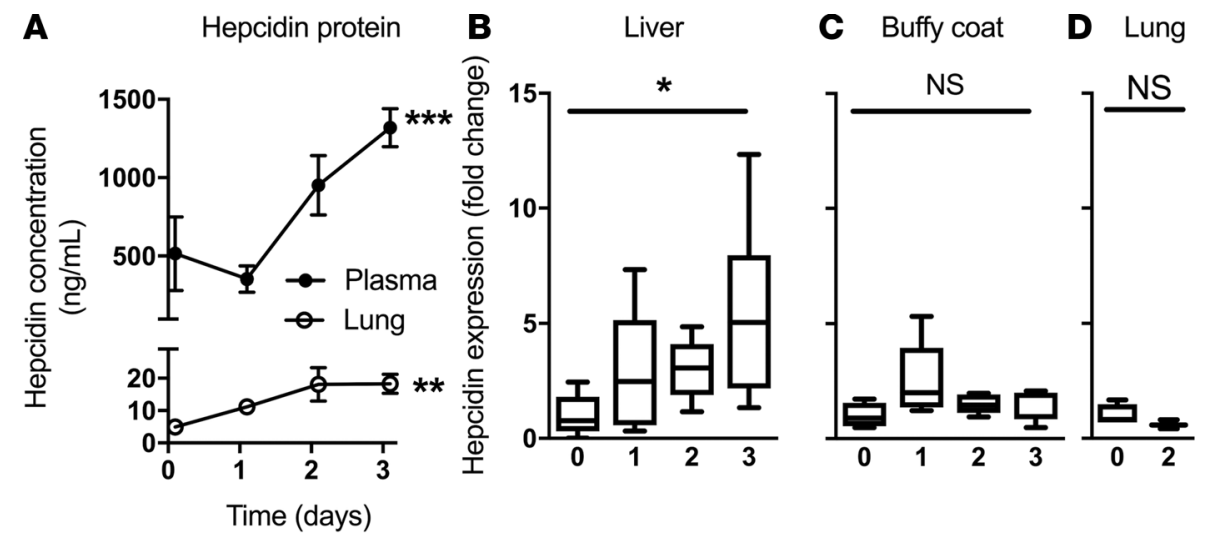

Figure 2. Hepcidin induction during Klebsiella pneumonia. Time 0 represents naive animals. (A) Hepcidin protein levels in the plasma and whole lung homogenate following infection. Data represent mean \pm SEM. (B-D) Hepcidin mRNA in the liver, buffy coat, and lung normalized to GAPDH expression and then to day 0. Box and whisker plots show median (line within box), upper and lower quartiles (upper and lower box boundaries), and total range (bars); $n=4-6$ animals per time point in each panel; ${ }^{*},{ }^{* *}$, and ${ }^{* * *}$ denote $P$ values of $<0.05,<0.01$, and $<0.001$, respectively (1-way ANOVA, multiple comparison for linear trend for panels $\mathbf{A}-\mathbf{C}, t$ test for panel $\mathbf{D}$ ).

Increased iron availability worsens the outcome of infection. To determine whether iron availability influences host defense during the course of infection, we tested the effect of transient increase in extracellular iron availability, achieved by i.p. injection of ferric ammonium citrate, on the outcome of Klebsiella pneumonia. Mice treated with iron prior to intrapulmonary K. pneumoniae challenge developed transient elevation in plasma iron that resolved completely within 12 hours (Figure 1D) without influencing lung iron (Supplemental Figure 1; supplemental material available online with this article; https://doi.org/10.1172/jci. insight.92002DS1), consistent with prior studies indicating that excess iron is rapidly cleared from the blood and redistributed to the BM, spleen, and liver (6). This transient increase in iron availability during the first 24 hours after infection was associated with a markedly increased mortality from pneumonia associated with increased bacterial burden in the lung and the blood (Figure 1, E and F). Since WT mice developed hypoferremia upon infection with $K$. pneumoniae, and excess iron was associated with poor infection outcome, we hypothesized that endogenous iron regulation was important for controlling infection.

Hepcidin regulates plasma iron during bacterial pneumonia by an IL-6-dependent mechanism. To assess the role of hepcidin in mediating hypoferremia during pneumonia, we began by characterizing hepcidin expression during the infection. Infection resulted in a marked increase in hepcidin protein levels in plasma, in parallel with the development of hypoferremia (Figure 2A). Hepcidin protein concentration also increased in the

A

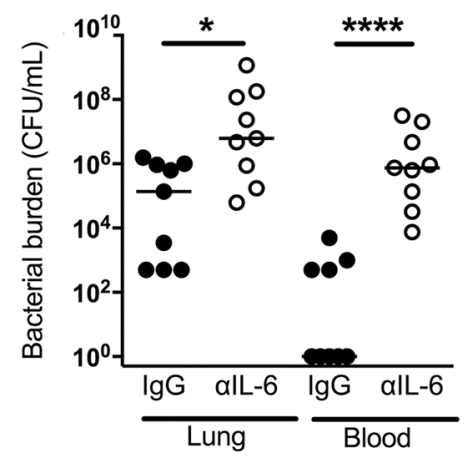

B

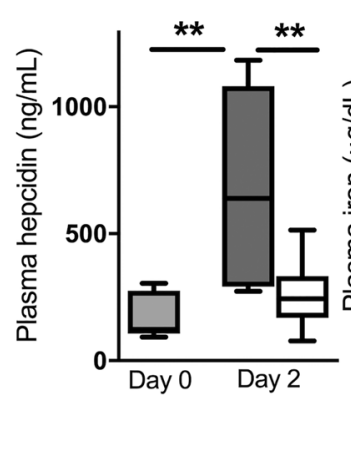

C

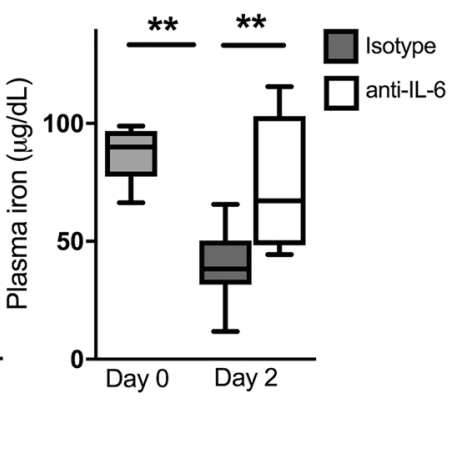

Figure 3. The role of IL-6 in hepcidin induction during Klebsiella pneumonia. (A) Animals were treated daily with an isotype control or IL-6 neutralizing monoclonal antibody and assessed for bacterial burden on day 2 of infection. Horizontal lines represent median, and each circle represents 1 animal; animals with no detectable bacteria are reported to have a bacterial burden of 1 CFU on the logarithmic scale; * and ${ }^{* * *}$ denote $P$ values of $<0.05$ and $<0.0001$ by Mann-Whitney $U$ test, respectively. (B and C) Plasma hepcidin protein and iron levels in uninfected and infected animals treated with isotype or IL-6 neutralizing antibody. Box and whisker plots show median (line within box), upper and lower quartiles (upper and lower box boundaries), and total range (bars) combined from 2 experiments; $n=8-9$ animals per group; ${ }^{* *} P<0.01$, one way ANOVA Tukey post-test. 
A

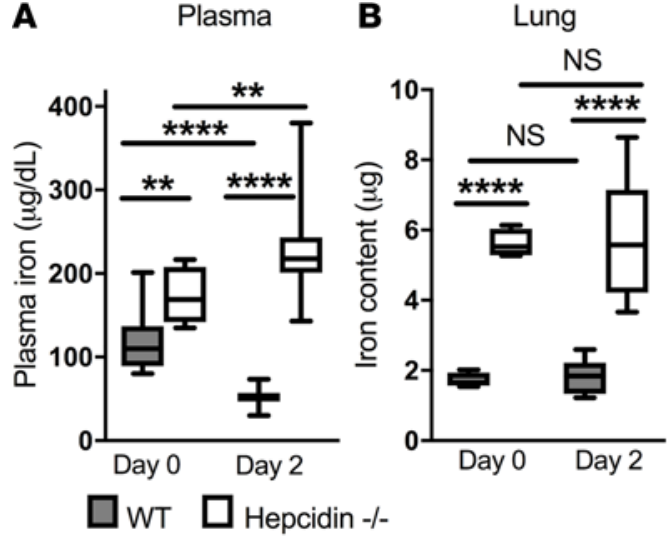

C Survival

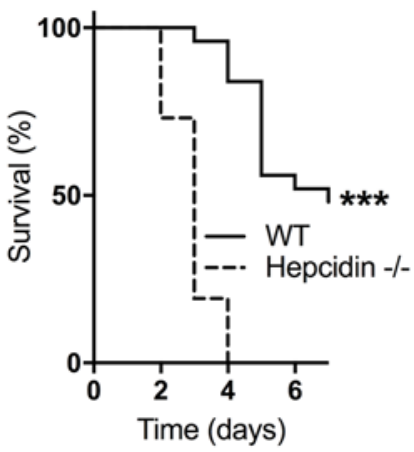

D

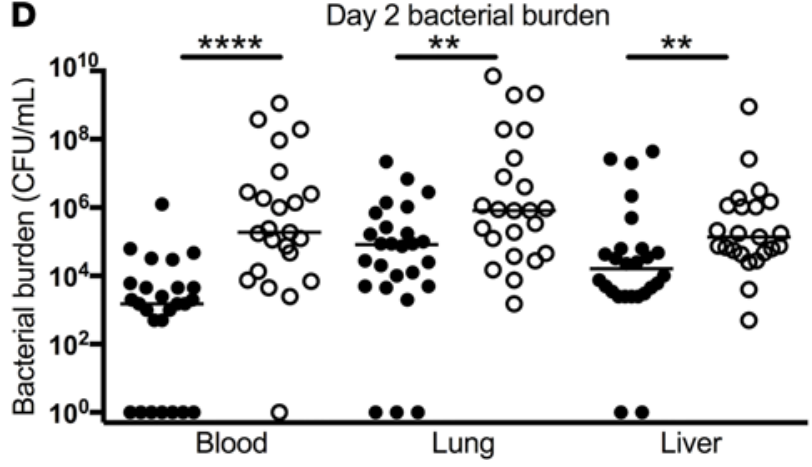

Figure 4. The role of hepcidin in Klebsiella pneumonia and dissemination. (A and $\mathbf{B}$ ) Comparison of plasma or lung nonheme iron levels in WT and hepcidin ${ }^{-/-}$mice before and 2 days after infection. Box and whisker plots show median (line within box), upper and lower quartiles (upper and lower box boundaries), and total range (bars) of data from 2 experiments; $n=4-9$ per group; ${ }^{* *}$ and ${ }^{* * * *}$ denote $P$ values of $<0.01$ and $<0.0001$ by one-way ANOVA with Tukey post test, respectively. (C) Survival of WT and hepcidin ${ }^{-/-}$mice during pneumonia. $n=26-30$ mice per group, combined from 3 independent experiments; ${ }^{* *} P<0.001$ Mantel-Cox test. (D) Bacterial burden on day 2 of infection in WT and hepcidin ${ }^{-/-}$mice in indicated organs. Pooled data from 3 independent experiments; $n=23-26$. Horizontal lines represent median and each circle represents one animal; animals with no detectable bacteria are reported to have a bacterial burden of 1 CFU on the logarithmic scale; ${ }^{* *}$ and ${ }^{* * *}$ denote $P$ values of $<0.01$ and $<0.0001$, respectively, using the Mann-Whitney $U$ test.

lung but at relatively low concentrations $(\sim 15 \mathrm{ng}$ in the entire lung, as compared with $\sim 1,500 \mathrm{ng} /$ $\mathrm{ml}$ of plasma on day 3; Figure 2A). The liver is the dominant source of hepcidin at baseline $(17,18)$, but hepcidin can be induced in leukocytes and airway epithelial cells in response to inflammatory stimuli under in vitro conditions (19-21). We thus measured hepcidin transcription in the liver, blood,

and lungs during infection and found a robust induction of hepcidin mRNA in the liver (Figure 2B) but not in peripheral blood leukocytes or lungs (Figure 2, C and D), suggesting that liver is the main source of hepcidin during the infection and elevations in lung hepcidin protein likely represent extravasation from the blood.

The cytokine IL- 6 is a key inducer of acute phase proteins in the liver and an important regulator of hepcidin expression in vivo (9). IL-6 is induced in the lungs in this model of pneumonia (22). We found that immunoneutralization of IL- 6 resulted in higher blood and lung bacterial burden 2 days following infection (Figure 3A). In addition, IL-6 neutralization abrogated the increase in plasma hepcidin during the infection and eliminated the associated hypoferremia (Figure 3, B and C), indicating that hepcidin induction during pneumonia is dependent on IL-6.

Hepcidin mediates hypoferremia during bacterial pneumonia and is required for survival. Reduced availability of plasma iron in the context of acute inflammation can occur as a result of both hepcidin-dependent and -independent mechanisms (23-25). We therefore tested the contribution of hepcidin in regulation of plasma iron levels in the context of bacterial pneumonia. In contrast to reduction in plasma iron during infection in WT mice, plasma iron in hepcidin-deficient mice became more elevated during the infection (Figure 4A), indicating that hepcidin is essential for controlling plasma iron levels following infection. Consistent with reports of tissue iron overload in hepcidin-deficient mice $(18,26)$, we found elevated lung iron levels in hepcidin-deficient as compared with WT mice, but these values did not change over the infection period (Figure 4B).

We next assessed the contribution of hepcidin-mediated iron sequestration to host defense against $K$. pneumoniae and found that hepcidin-deficient animals were markedly more susceptible to bacterial pneumonia, demonstrating $100 \%$ mortality compared with $50 \%$ mortality in WT controls (Figure 4C), comparable to mice treated with exogenous iron during infection (Figure 1, D and E). Hepcidin-deficient mice exhibited 23\% greater incidence of bacteremia and $>100$-fold higher median blood bacterial concentration, as well as $\sim 10$-fold higher bacterial burden in the lungs and liver as compared with WT mice (Figure 4D).

Since the hepatocytes in hepcidin-deficient animals are iron loaded, we reasoned that the increase of iron in infected hepcidin ${ }^{-/}$mice could originate from greater release of iron from damaged hepatocytes. We therefore quantified alanine aminotransferase and aspartate aminotransferase in the blood of infected mice to assess liver damage and found neither enzyme to be elevated in hepcidin ${ }^{-/-}$as compared with WT mice in the context of infection (Supplemental Figure 2), arguing against disproportionate 
A

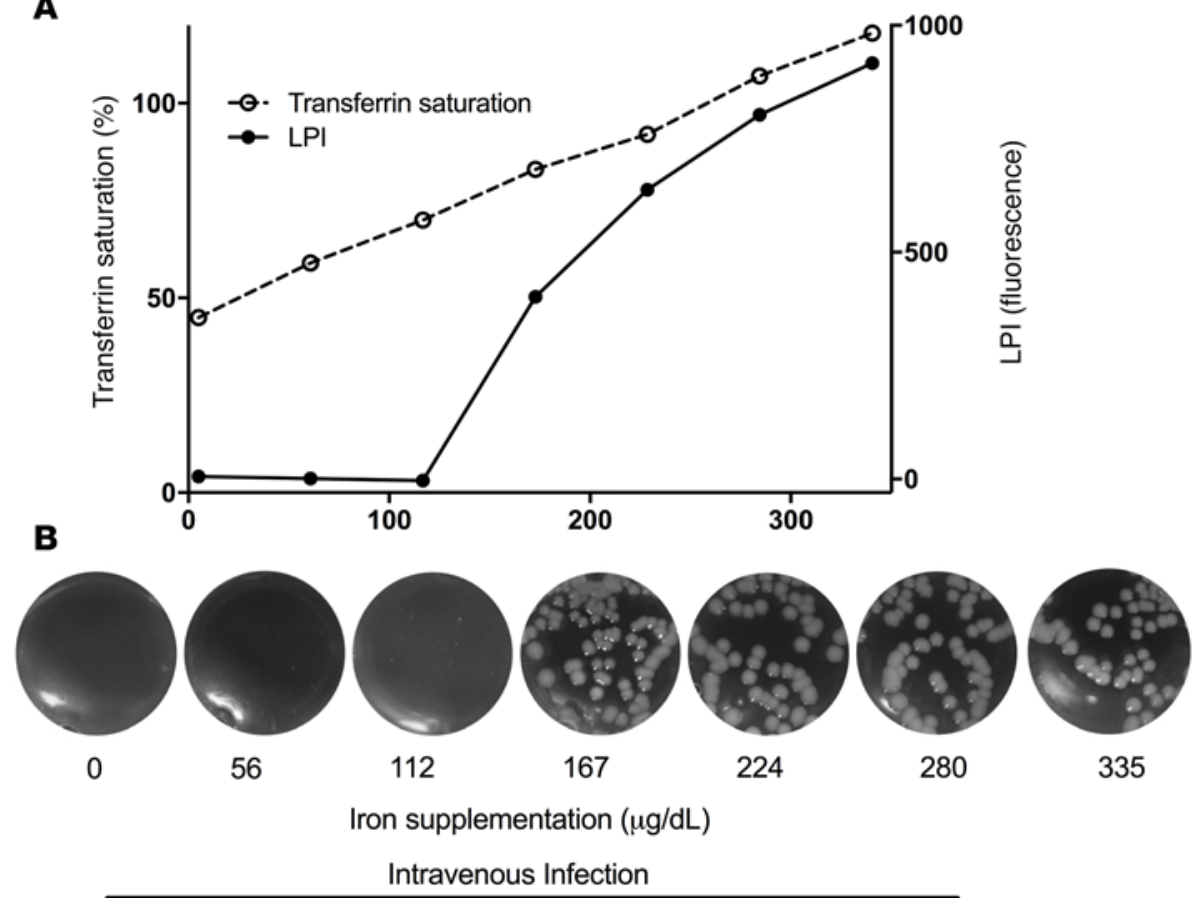

Figure 5. Effect of iron on Klebsiella growth in vitro and in vivo. (A) Human plasma was supplemented with ferric ammonium citrate and assayed for transferrin saturation and the presence of labile plasma iron (LPI). Transferrin saturation values of $>100 \%$ represent complete saturation of transferrin iron-binding sites in the presence of additional, unbound ferric iron ions. (B) Agar plates composed of $90 \%$ human plasma were supplemented with ferric ammonium citrate and inoculated with $K$. pneumoniae. Plates were monitored for occurrence of bacterial growth. Representative data from 3 independent plasma agar preparations. (C and D) Bacterial burden and plasma hepcidin protein in WT mice treated with either PBS or iron citrate 30 minutes prior to i.v. challenge with $K$. pneumoniae. Lungs and blood were harvested after 24 hours. Box and whisker plots show median (line within box), upper and lower quartiles (upper and lower box boundaries), and total range (bars). $n=11$ mice per group, combined form 2 experiments; ${ }^{*} P<0.05$, Mann-Whitney $U$ test; ${ }^{* *} P<0.01, t$ test.

hepatic injury as a contributor to worse outcomes in hepcidin-deficient hosts.

In order to assess whether the beneficial effects of hepcidin are dependent on iron, we used established protocols to induce severe iron-deficiency in hepcidin-deficient mice (27). We found that iron-depleted hepcidin ${ }^{-/-}$animals did not develop hyperferremia during infection, had lower body iron stores than WT mice, and were no more susceptible to infection compared with WT mice (Supplemental Figure 3, A and B). Taken together, these data suggest that the hepcidin-mediated control of bacterial growth during infection is iron dependent.

Excess iron promotes the growth of $K$. pneumoniae in plasma. To assess whether the increased susceptibility of hepcidin-deficient mice to infection was attributable to increased bacterial growth in the high-iron environment of the plasma, we assessed the effect of iron supplementation on the in vitro growth of $K$. pneumoniae in human plasma. Supplementing agar composed of $90 \%$ human plasma with ferric ammonium citrate at physiologically relevant concentrations incrementally raised the transferrin saturation of the sample, resulting in the appearance of non-transferrin-associated labile pool iron (Figure 5A). The appearance of labile pool iron at transferrin saturation greater than $70 \%$ is consistent with prior reports ( 28 , 29). K. pneumoniae growth was suppressed in plasma but was permitted by iron supplementation, consistent with reports that transferrin inhibits bacterial growth (30) (Figure 5B). Interestingly, we found that, in plasma preparations from 3 independent donors, addition of ferric iron resulted in $K$. pneumoniae out-growth coincided with the appearance of labile iron in the plasma, rather than with the total amount of iron citrate added. These data suggest that ferric ammonium citrate supports $K$. pneumoniae growth in plasma by providing iron, rather than acting as a carbon or nitrogen source.

We next sought to test the hypothesis that, during in vivo infection, elevated plasma iron is sufficient to enhance growth of blood-borne bacteria. To test this, we subjected WT mice with an i.p. injection of PBS or ferric ammonium citrate prior to i.v. challenge with $K$. pneumoniae. After 24 hours, mice treated with ferric ammonium citrate had higher blood bacterial content than mice treated with PBS, indicating that 

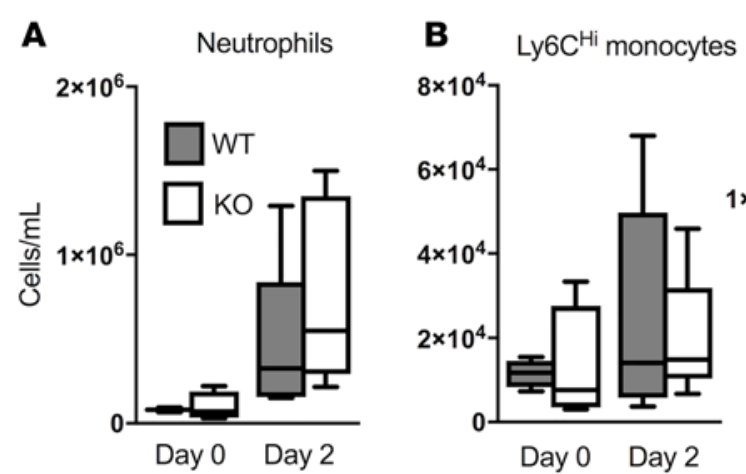

C Ly6C $^{10}$ monocytes

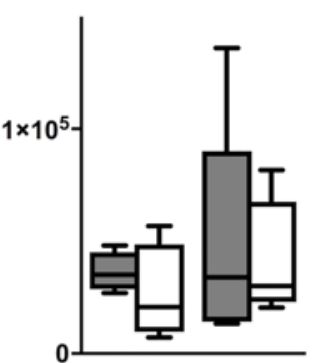

BAL
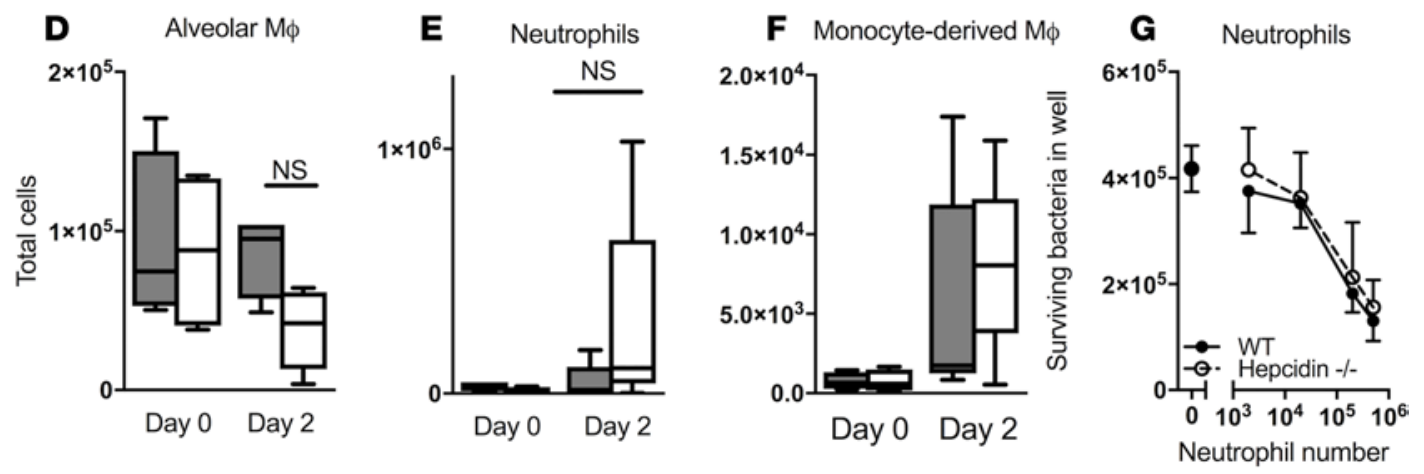

Figure 6. Leukocytes responses during Klebsiella pneumonia. (A-F) BAL and blood were harvested from WT and hepcidin ${ }^{-/-}$mice before and 2 days following infection. Box and whisker plots show median (line within box), upper and lower quartiles (upper and lower box boundaries), and total range (bars). $n=4-5$ per group, one way ANOVA Tukey post-test. No significant differences were found for any WT to hepcidin ${ }^{-1-}$ comparison. (C) Freshly isolated neutrophils from WT and hepcidin ${ }^{-/}$mice were coincubated with $2 \times 10^{5} \mathrm{CFU}$ of $K$. pneumoniae for 1 hour. Total surviving bacteria were enumerated from each well. $n=3$ independent experiments per group; data were analyzed using a 2-way ANOVA, and no differences were found between groups

elevated plasma iron directly mediates increased growth of $K$. pneumoniae in the blood stream (Figure 5C). Interestingly, mice treated with ferric ammonium citrate also had higher bacterial burdens in the lungs. We interpret this as evidence that, in mice with increased bacteremia, bacterial seeding of the lung from the bloodstream contributes to higher lung bacterial content. Similar to prior reports (31), mice treated with iron also had marked elevation of plasma hepcidin as compared with mice treated with PBS in response to acute iron loading (Figure 5D), indicating that hepcidin induction alone is not sufficient to protect mice from fatal infection if plasma iron levels are high and supporting the hypothesis that the beneficial effects of hepcidin are mediated via induction of hypoferremia, rather than an iron-independent effect.

Hepcidin-deficient mice have intact cellular immunity against Klebsiella. Iron deficiency and iron overload have complex effects on immune responses (32), and phagocytes from iron-overloaded hosts may have altered antibacterial properties that could contribute to impaired host defenses of hepcidin-deficient mice independent of the effects of acute hyperferremia on bacteria. In order to assess the functionality of the innate immune response in hepcidin-deficient mice, we analyzed leukocyte populations in the blood and BAL (Figure 6, A-F). We found similar concentrations of circulating neutrophils, and Ly6C $\mathrm{C}^{\text {hi }}$ and Ly $6 \mathrm{C}^{\text {lo }}$ monocytes in WT and hepcidin-deficient mice at baseline and 2 days following infection. The number of BAL alveolar macrophages, neutrophils, and monocyte-derived macrophages were also similar between WT and hepcidin-deficient hosts, both at baseline and following infection, arguing against a defect in leukocyte quantity or recruitment in the absence of hepcidin. Since oxidative burst is an iron-dependent process, we compared the generation of reactive oxygen species in lung and blood leukocyte populations and found no difference between infected WT and hepcidin-deficient mice (Supplemental Figure 4). Similarly, neutrophils from WT and hepcidin-deficient mice exhibited comparable ex vivo bacterial killing (Figure 6G). While these data do 
A

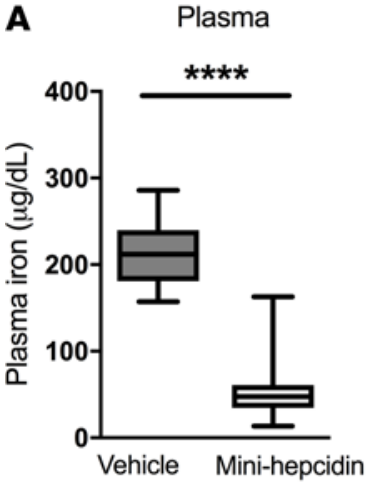

B

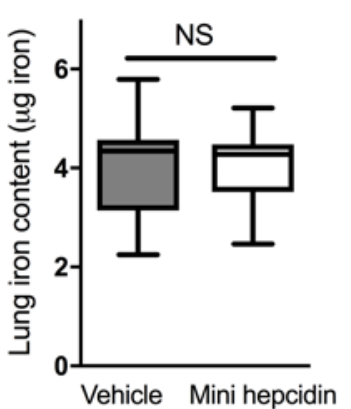

C

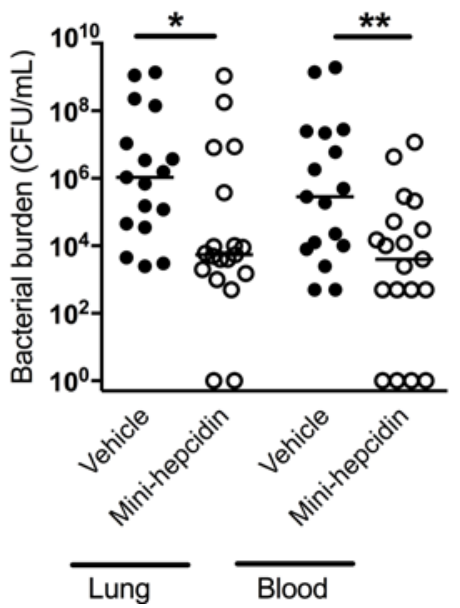

D Survival

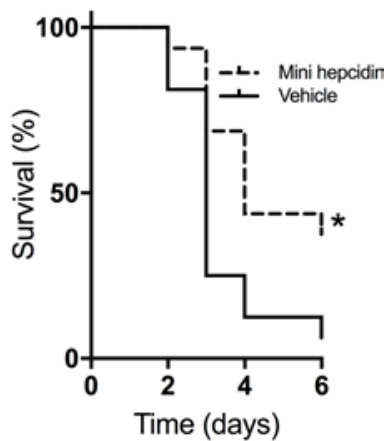

Figure 7. The effect of hepcidin agonist treatment in Klebsiella pneumonia in hepcidin-deficient mice. (A and B) Nonheme iron levels in the plasma and lung of vehicle-treated and minihepcidin-treated hepcidin-deficient mice. Box and whisker plots show median (line within box), upper and lower quartiles (upper and lower box boundaries), and total range (bars). $n=17-19$ per group combined from 2 independent experiments; ${ }^{* * *} P<0.001, t$ test. (C) Bacterial burden in blood and lungs. Horizontal lines represent median and each circle represents 1 animal; animals with no detectable bacteria are reported to have a bacterial burden of 1 CFU on the logarithmic scale; $n=17-19$ per group, combined data from 2 experiments; ${ }^{*}$ and ${ }^{* *}$ denote $P$ values $<0.05$ and $<0.01$ by Mann-Whitney $U$ test, respectively. (D) Survival of hepcidin-deficient mice treated with either vehicle or hepcidin analogue days $0-5 ; n=16$ per group, combined result of 2 experiments; ${ }^{*} P<0.05$ by Mantel-Cox test.

not formally exclude the possibility that hepcidin deficiency impairs the immune system in some other way, they suggest intact cellular innate immune mechanisms against $K$. pneumoniae in hepcidin ${ }^{-/-}$mice.

Restoring hypoferremia is sufficient to protect hepcidin-deficient mice from infection. Impaired hepcidin production is common in the clinical setting - for example, in patients with liver disease (33-36) — and our data suggests a mechanism by which these hosts may be predisposed to Gram-negative bacterial infections. As proof-of-principle of a potential therapeutic approach in this population, we assessed the effect of minihepcidin, a hepcidin analogue, on the outcome of $K$. pneumoniae infection in hepcidin-deficient hosts. We used a previously described synthetic peptide based on the $9 \mathrm{~N}$-terminal amino acids of the hepcidin molecule, which are necessary and sufficient to induce the degradation of ferroportin $(37,38)$. Daily treatment with the hepcidin analogue beginning on the day of infection resulted in markedly lowered plasma iron levels in infected hepcidin-deficient mice as compared with vehicle-treated controls without affecting the total lung iron content (Figure 7, A and B), consistent with utilization of plasma iron in erythropoiesis (6). The treatment also resulted in $>99 \%$ reduction in median lung and blood bacterial burden in infected hepcidin-deficient mice and 40\% reduction in mortality compared with vehicle alone (Figure 7, C and D). These data provide evidence that the acute induction of hypoferremia during infection in hepcidin-deficient hosts may represent a plausible therapeutic strategy.

\section{Discussion}

Although the competition for iron is thought to be a general feature of antimicrobial host response, the mechanisms and contribution of iron restriction in most infections are undefined. In particular, hepcidin-mediated hypoferremia has only been implicated in host defense against $V$. vulnificus, an organism with specific affinity for iron-overloaded hosts, but its role in defense against common pathogens and in normal hosts is not known (10). We showed that hepcidin was necessary for protection against bacterial dissemination in Gram-negative pneumonia and that treatment with iron citrate was sufficient to permit bacterial outgrowth in WT mice. Finally, we showed that treatment with minihepcidin restored hypoferremia and rescued iron-overloaded, hepcidin-deficient hosts from the infection. Taken together, these data suggest that hepcidin-mediated iron sequestration protects against Gram-negative pneumonia.

Our data suggest that the hepcidin-dependent protection was mediated by reducing plasma iron, as indicated by our observation that WT mice treated with iron citrate were susceptible to K. pneumoniae infection despite robust hepcidin induction (Figure 1, E and F) and that hepcidin-deficient mice were protected from 
infection if starved of iron (Supplemental Figure 3B). While there may be iron-independent effects of ferric ammonium citrate administration that we have not accounted for, such as bacterial fermentation of citrate, we are encouraged that the phenotype of iron citrate-treated mice is remarkably similar to that of hepcidin ${ }^{-/}$ mice in terms of death and bacterial burden. Furthermore, we found that hepcidin-deficient mice treated with minihepcidin were robustly protected from infection despite having tissue iron overload, suggesting that restriction of iron in the plasma is sufficient for protection (Figure 7, C and D). Hepcidin exhibits antimicrobial activity against a broad array of pathogens in vitro, and an iron-independent direct microbicidal role for hepcidin has thus been suggested $(17,39,40)$. Arguing against such a direct microbicidal role, we report plasma hepcidin levels at concentrations much lower than those required to mediate killing in vitro (39). Similarly, administration of a hepcidin analogue, which lacks the $\beta$-defensin motif required for its antimicrobial activity (37), rescued the phenotype of hepcidin-deficient mice (Figure 7, C and D).

Although hepcidin-independent mechanisms of hypoferremia have been reported after challenge with inflammatory stimuli $(23,25,41)$, we found that hypoferremia during pneumonia was completely abrogated in hepcidin-deficient mice, and in fact, plasma iron levels became elevated following infection in hepcidin-deficient mice. Our use of an infectious agent, rather than sterile inflammatory stimuli used in other reports, may account for this difference. We speculate that this increase in plasma iron levels reflects decreases in iron consumption by cytokine-suppressed erythropoiesis (24) and release of iron from intracellular sources damaged by infection, such as the liver (42). We thus propose a model in which infections result in a tendency toward increased extracellular iron that benefits the pathogen, and the protective role of inflammation-mediated hepcidin induction is to counteract this phenomenon. Furthermore, while hepcidin can be produced by leukocytes, airway epithelial cells and other tissues(19-21), our results indicate that hepcidin is predominantly produced by the liver and circulates systemically in the context of bacterial pneumonia. Consistent with this, we observed that hepcidin induction was dependent on IL-6, similar to observations with other systemic inflammatory stimuli (43). In addition, we found that IL-6 neutralization disrupted host defense against $K$. pneumoniae (Figure 3A), in corroboration with previous reports (22). While IL-6 induces many acute phase reactants, such as $C$ reactive protein, which are crucial to the innate immune response, we speculate that hepcidin induction may be a component of IL-6-mediated protection.

These data provide the host counterpart to the existing literature on the role of the bacterial siderophore system in the virulence of Gram-negative pathogens $(30,44)$. The hypervirulent strain of $K$. pneumoniae used in this study produces yersiniabactin, which supports bacterial growth in the respiratory tract, and glycosylated enterobactin, which promotes bacterial growth in the bloodstream by evading lipocalin-2, a host-derived molecule that sequesters nonglycosylated enterobactin and limits microbial iron uptake (45-49). Our findings indicate that even bacteria equipped with these high-affinity acquisition systems are susceptible to a host mechanism that limits iron availability in the host environment. As such, we speculate that these findings may be broadly applicable to infections caused by other phylogenetically related aerobic Gram-negative pathogens (50).

This work has several implications for future research. First, our results may have implications for the therapeutic use of hepcidin antagonists, which are currently under development for the treatment of anemia of inflammation. In this context, we posit that relieving the iron-restricted state could predispose some individuals to Gram-negative sepsis. Second, impaired hepcidin production is a feature of several common clinical entities, including alcohol intoxication and chronic liver disease (33-36), illnesses associated with striking susceptibility to Gram-negative bacteremia $(51,52)$. Our data suggest that impaired hepcidin production may represent an unappreciated mechanism predisposing these hosts to infection. Finally, hepcidin agonists may represent a novel therapeutic strategy in illnesses where hepcidin production is diminished, such as liver disease and hereditary hemochromatosis, and possibly more broadly in acquired iron-overload states.

\section{Methods}

Supplemental Methods are available online with this article.

Animals and in vivo procedures. C57BL/6 mice, purchased from the Jackson Laboratories. Hepc1 ${ }^{-1-}$ mice generated on a C57BL/ 6 background (26) - acquired from the University of California, Los Angeles, California, USA - were bred at the University of Virginia under specific-pathogen-free conditions for a minimum of 2 generations, and offspring were used in the experiments. To minimize any effect of differing microbiota between groups of animals, all animals were bred and maintained in the same room in the vivarium and used bedding from cages of breeders, and animals to be used in experiments were intermixed at weekly intervals. Age- and sex-matched male and female 6- to 12-week-old mice were used in experiments. 
Standard rodent chow has artifactually high iron content that causes near-maximal expression of hepcidin at baseline in WT animals (43). To correct this, all animals were conditioned on a low-iron diet (2-4 ppm iron; Teklad diet TD.80396, Envigo) for 5-7 days prior to, and throughout the duration of, all experiments. In our pilot studies and published reports $(43,53)$, this protocol normalizes baseline hepcidin expression without inducing iron deficiency.

Experimental bacterial pneumonia was induced as previously described $(15,16)$, by intratracheal inoculation of 500-1,500 CFU of K. pneumoniae strain 43816 (American Type Culture Collection). In some experiments, 1,500 CFU of K. pneumoniae was delivered i.v. in $100 \mu 1$ PBS. To induce transient hyperferremia without iron overload, $900 \mu \mathrm{g}$ of ferric ammonium citrate in $100 \mu 1$ PBS was delivered i.p., using a previously characterized protocol $(54,55)$. In some experiments, mice were given $1 \mathrm{mg}$ anti-IL-6 antibody or isotype control i.p. 3 hours prior to and 24 hours following infection (clones MP5-20F3 and HRPN1, BioXcell). In other experiments, $100 \mathrm{nmol}$ of the synthetic hepcidin analogue PR73 (manufactured in house), referred to as minihepcidin in text, or vehicle was administered via i.p. injection beginning 5 hours prior to infection and then every 24 hours until day 5 after infection. PR73 was synthesized using Fmoc solid-phase peptide synthesis and was solubilized for in vivo use with SL220 (a polyethylene glycosylated-phospholipid based solubilzer; NOF America Corporation) as previously described (11, 37, 38).

Tissue harvest and determination of bacterial burden. Animals were euthanized with an overdose of a ketamine/xylazine solution, blood was collected from the right ventricle into heparinized syringes, and pulmonary vasculature was perfused with $2 \mathrm{ml}$ of $2 \mathrm{mM}$ EDTA solubilized in PBS. The median liver lobe was collected for RNA analysis. Lungs and liver were homogenized in $1 \mathrm{ml}$ PBS. BAL was performed by serially inflating the lungs via an intratracheal catheter with $1 \mathrm{ml}$ of $2 \mathrm{mM}$ EDTA solubilized in PBS and recovering the fluid 4 times (56). For bacterial enumeration, lung homogenates and blood were serially diluted in sterile water and cultured on blood agar plates, as previously described (57).

$R N A$, protein, and iron analysis. Tissue RNA was extracted using TRIzol and chloroform (Thermo-Fisher Scientific). Buffy coat RNA was extracted using a commercial kit (Qiagen). A commercial kit was used to synthesize cDNA (Promega). Hepcidin transcript was quantified by quantitative PCR in duplicate using SYBR green (Bioline). Expression was calculated using the $\Delta \Delta \mathrm{C}(\mathrm{t})$ method normalized to glyceraldehyde 3-phosphate dehydrogenase, and then relative to hepcidin expression of uninfected animals. Primers were custom synthesized (Sigma Aldrich) as previously published (35): hepcidin 5' TGCAGAAGAGAAGGAAGAGAGACA 3' (forward) and 5' CACACTGGGAATTGTTACAGCATT 3' (reverse); glyceraldehyde 3-phosphate dehydrogenase 5' TTCACCACCATGGAGAAGGC 3' (forward) and 5' GGCATGGACTGTGGTCATGA 3' (reverse) (58). The thermal cycling conditions were as follows: 2 minutes at $95^{\circ} \mathrm{C}, 10$ minutes at $95^{\circ} \mathrm{C}, 5$ seconds at $95^{\circ} \mathrm{C}$, and 10 seconds at $55^{\circ} \mathrm{C}$ for 40 cycles. Reactions were performed on a BioRad iQ5 Cycler. Protein concentration of hepcidin was quantified using a commercial ELISA kit (Intrinsic Life Sciences) according to the manufacturer instructions. Plasma iron was quantified with a commercial kit (Sekisui Diagnostics) according to manufacturer instructions with minor modifications - namely, samples were scaled to volume and centrifuged at $10,000 \mathrm{~g}$ for 10 minutes between steps to clear insoluble material. Transferrin saturation was determined by measuring the unsaturated iron binding capacity (UIBC) of each sample using a commercial kit (Sekisui Diagnostics). UIBC values were added to plasma iron values in each sample to calculate the total iron binding capacity (TIBC). Transferrin saturation was calculated as (plasma iron/TIBC) $\times 100 \%$.

Tissue heme and nonheme iron levels were quantified using the ferrozine assay, as previously described (59-61). Whole lungs and left lateral lobe of the liver were homogenized in $1 \mathrm{ml}$ of water. BAL was collected into $1 \mathrm{ml}$ of $2 \mathrm{mM}$ EDTA solubilized in PBS. Briefly, $100 \mu 1$ sample were heated in $100 \mu 1$ buffer containing $1 \mathrm{~N}$ hydrochloric acid and 10\% trichloroacetic acid with (total iron) or without (nonheme iron) $2.25 \% \mathrm{KMnO}_{4}$ at $60^{\circ} \mathrm{C}$ for 2 hours. Samples were centrifuged at $16,000 \mathrm{~g}$ for 10 minutes to remove insoluble material and were incubated with $50 \mu$ iron detection reagent $(6.5 \mathrm{mM}$ ferrozine, $6.5 \mathrm{mM}$ neucoproine, $1 \mathrm{M}$ L-ascorbic acid, 2.5 M ammonium acetate) for 10 minutes and then plated in duplicate and read at $\mathrm{A}_{590} \mathrm{~nm}$ on a Dynex Triad microplate reader (Dynex Technologies). Heme-iron values were calculated by subtracting nonheme iron values from total iron content. Labile plasma iron (LPI), the major component of non-transferrin-bound iron, was measured using a published protocol with minor modifications (62): briefly, HEPES buffered saline was iron-depleted by incubation with Chelex-100 chelating resin (Bio-Rad) for 1 hour, filtered, and supplemented with $60 \mathrm{nM}$ deferrioxamine (Sigma-Aldrich). For LPI measurements, $10 \mu \mathrm{l}$ of plasma samples were mixed with either $140 \mu \mathrm{l}$ of $\mathrm{HBS} / 50 \mu \mathrm{M}$ dihydrorhodamine/ $40 \mu \mathrm{M}$ ascorbic acid/0.5 $\mathrm{mM}$ nitriloacetic acid (-DFP) or $140 \mu \mathrm{l}$ of the same buffer supplemented with $100 \mu \mathrm{M}$ deferriprone (+DFP). 
Changes in fluorescence were measured over 40 minutes, and the slope was calculated for each sample. The difference between -DFP and +DFP was used as a measure of LPI. The experiment was repeated a total of 3 times using different plasma donors with similar results.

Flow cytometry. Cell suspensions from blood and BAL fluid were prepared and analyzed by flow cytometry as previously described, with minor modifications $(16,63,64)$. The following antibodies were used for surface antigen staining: anti-MHC-II APC (clone M5/114.15.2), anti-CD11b Pe-Cy7 (clone M170), and anti-CD115 APC (clone AF598) from eBioscience; anti-CD11c PerCP (clone HL3) and anti-CD64 brilliant violet 421 (clone X54-5/7.1) from BioLegend; and anti-CD16/CD32 (clone 2.4G2), anti-Ly6G $\mathrm{PE}$ (clone 1A8), and anti-CD45 AmCyan (clone 30-F11) from BD Biosciences. After extracellular staining, cells were washed in 1\% FCS in PBS once and resuspended in the same buffer. Data were acquired on a FACS Canto II instrument using BD FACS Diva software (version 8.0; BD Biosciences) and analyzed with FlowJo software (version 8.8.6; Tree Star Inc.). The absolute cell number was calculated as the product of the cell type frequency (as determined by gating analysis) and the total number of cells in the sample (as determined by manually counting under a hemocytometer).

In vitro experiments. BM neutrophils were isolated by grinding the tibia, femur, pelvis, and spine from uninfected mice in a sterile mortar and pestle as previously described $(15,57)$, followed by positive selection using anti-Ly6G magnetic beads, according to the manufacturer's instructions (MACS; Miltenyi Biotec). Isolated populations were found to have $>90 \%$ viability by examination in trypan blue. Neutrophil bacterial killing was quantified using a published protocol with minor modifications (57). Briefly, K. pneumoniae was opsonized at a concentration of $8 \times 10^{6} \mathrm{CFU} / \mathrm{ml}$ in $20 \%$ fresh mouse serum and HBSS for 30 minutes at $37^{\circ} \mathrm{C}$, washed once in cold water, once in HBSS, and resuspended in HBSS. Aliquots of $2 \times 10^{5}$ bacteria were added to sterile U-bottom 96-well plates and coincubated with $5 \times 10^{5}, 2 \times 10^{5}, 2 \times 10^{4}, 2 \times 10^{3}$, or no neutrophils from WT or hepcidin-deficient mice in triplicate in a total volume of $200 \mu \mathrm{HBSS}$. Samples were incubated in a humidified chamber with $5 \% \mathrm{CO}_{2}$ at $37^{\circ} \mathrm{C}$ for 1 hour. Number of surviving bacteria was determined by hypotonic lysis of neutrophils in cold water and serial dilution and culture.

Human plasma was inactivated $\left(30 \mathrm{~min}\right.$ at $\left.56^{\circ} \mathrm{C}\right)$ and incubated overnight at $37^{\circ} \mathrm{C}$ with $0-60 \mu \mathrm{M}$ ferric ammonium citrate (Sigma-Aldrich), followed by quantification of transferrin saturation and LPI. The samples were then used to generate $90 \%$ plasma agar solid media, poured in sterile 12 -well tissue culture plates. $K$. pneumoniae was suspended at $1 \times 10^{4} \mathrm{CFU} / \mathrm{ml}$ and $5 \mu \mathrm{l}$ plated in triplicates on plasma agar wells and incubated for 24 hours at $37^{\circ} \mathrm{C}$; bacterial colonies were counted and photographed.

Statistics. Data were analyzed using Prism software (version 6.0, GraphPad Software). The Mantel-Cox test was used to analyze survival data. A one-way ANOVA with a post-test for linear trend was used to analyze change in a single parameter over time, and one-way ANOVA with a Tukey multiple comparison post-test was used to analyze differences between 3 or more groups. A 2-way ANOVA with Tukey multiple comparison post-test was used to compare changes over time between 2 groups. An unpaired, 2-tailed $t$ test was used to compare 2 groups for RNA, protein, and iron levels. A Mann-Whitney $U$ test was used to compare bacterial burden between groups. $P<0.05$ was considered statistically significant.

Study approval. Animal experiments were performed in compliance with NIH Guide for the Care and Use of Laboratory Animals, The US Animal Welfare Act, and PHS Policy on Humane Care and Use of Laboratory Animals and was approved by the Institutional Animals Care and Use Committee at the University of Virginia (protocol 3571)

\section{Author contributions}

$\mathrm{KRM}, \mathrm{EN}, \mathrm{TG}$, and BM conceived the project, designed experiments, and interpreted data; KRM, ZZ, AMB, REC, DS, and MDB performed experiments; KRM and DS generated the figures; SV, EN, and TG designed and contributed materials; and KRM and BM wrote the first draft of the manuscript. All authors revised the manuscript.

\section{Acknowledgments}

This work was supported by NIH grants R21AI117397 and R01HL098329-05 awarded to BM; and R01DK065029 awarded to TG. The funders had no role in study design, data collection and analysis, decision to publish, or preparation of the manuscript.

Address correspondence to: Borna Mehrad, P.O. Box 800546, Charlottesville, Virginia 22908, USA. Phone: 434.243.4845; E-mail: mehrad@virginia.edu. 
1. Gaynes R, Edwards JR, National Nosocomial Infections Surveillance System. Overview of nosocomial infections caused by gram-negative bacilli. Clin Infect Dis. 2005;41(6):848-854.

2. Meatherall BL, Gregson D, Ross T, Pitout JD, Laupland KB. Incidence, risk factors, and outcomes of Klebsiella pneumoniae bacteremia. Am J Med. 2009;122(9):866-873.

3. Uslan DZ, et al. Age- and sex-associated trends in bloodstream infection: a population-based study in Olmsted County, Minnesota. Arch Intern Med. 2007;167(8):834-839.

4. Skaar EP. The battle for iron between bacterial pathogens and their vertebrate hosts. PLoS Pathog. 2010;6(8):e1000949.

5. Cassat JE, Skaar EP. Iron in infection and immunity. Cell Host Microbe. 2013;13(5):509-519.

6. Craven CM, Alexander J, Eldridge M, Kushner JP, Bernstein S, Kaplan J. Tissue distribution and clearance kinetics of non-transferrin-bound iron in the hypotransferrinemic mouse: a rodent model for hemochromatosis. Proc Natl Acad Sci USA. 1987;84(10):3457-3461.

7. Nemeth E, et al. Hepcidin regulates cellular iron efflux by binding to ferroportin and inducing its internalization. Science. 2004;306(5704):2090-2093.

8. Besson-Fournier C, et al. Induction of activin B by inflammatory stimuli up-regulates expression of the iron-regulatory peptide hepcidin through Smad1/5/8 signaling. Blood. 2012;120(2):431-439.

9. Nemeth E, Valore EV, Territo M, Schiller G, Lichtenstein A, Ganz T. Hepcidin, a putative mediator of anemia of inflammation, is a type II acute-phase protein. Blood. 2003;101(7):2461-2463.

10. Michels K, Nemeth E, Ganz T, Mehrad B. Hepcidin and Host Defense against Infectious Diseases. PLoS Pathog. 2015;11(8):e1004998.

11. Arezes J, et al. Hepcidin-induced hypoferremia is a critical host defense mechanism against the siderophilic bacterium Vibrio vulnificus. Cell Host Microbe. 2015;17(1):47-57.

12. Khan FA, Fisher MA, Khakoo RA. Association of hemochromatosis with infectious diseases: expanding spectrum. Int $J$ Infect Dis. 2007;11(6):482-487.

13. Agbaht K, et al. Bacteremia in patients with ventilator-associated pneumonia is associated with increased mortality: A study comparing bacteremic vs. nonbacteremic ventilator-associated pneumonia. Crit Care Med. 2007;35(9):2064-2070.

14. Magret $\mathrm{M}$, et al. Bacteremia is an independent risk factor for mortality in nosocomial pneumonia: a prospective and observational multicenter study. Crit Care. 2011;15(1):R62.

15. Barletta KE, Cagnina RE, Burdick MD, Linden J, Mehrad B. Adenosine A(2B) receptor deficiency promotes host defenses against gram-negative bacterial pneumonia. Am J Respir Crit Care Med. 2012;186(10):1044-1050.

16. Bettina A, et al. M-CSF Mediates Host Defense during Bacterial Pneumonia by Promoting the Survival of Lung and Liver Mononuclear Phagocytes. J Immunol. 2016;196(12):5047-5055.

17. Park CH, Valore EV, Waring AJ, Ganz T. Hepcidin, a urinary antimicrobial peptide synthesized in the liver. J Biol Chem. 2001;276(11):7806-7810.

18. Zumerle S, et al. Targeted disruption of hepcidin in the liver recapitulates the hemochromatotic phenotype. Blood. 2014;123(23):3646-3650.

19. Armitage AE, Pinches R, Eddowes LA, Newbold CI, Drakesmith H. Plasmodium falciparum infected erythrocytes induce hepcidin (HAMP) mRNA synthesis by peripheral blood mononuclear cells. Br J Haematol. 2009;147(5):769-771.

20. Chen QX, et al. Silencing airway epithelial cell-derived hepcidin exacerbates sepsis induced acute lung injury. Crit Care. 2014;18(4):470

21. Nguyen NB, Callaghan KD, Ghio AJ, Haile DJ, Yang F. Hepcidin expression and iron transport in alveolar macrophages. Am J Physiol Lung Cell Mol Physiol. 2006;291(3):L417-L425.

22. Sutherland RE, Olsen JS, McKinstry A, Villalta SA, Wolters PJ. Mast cell IL-6 improves survival from Klebsiella pneumonia and sepsis by enhancing neutrophil killing. J Immunol. 2008;181(8):5598-5605.

23. Guida C, et al. A novel inflammatory pathway mediating rapid hepcidin-independent hypoferremia. Blood. 2015;125(14):2265-2275.

24. Langdon JM, et al. Hepcidin-dependent and hepcidin-independent regulation of erythropoiesis in a mouse model of anemia of chronic inflammation. Am J Hematol. 2014;89(5):470-479.

25. Armitage AE, et al. Induced Disruption of the Iron-Regulatory Hormone Hepcidin Inhibits Acute Inflammatory Hypoferraemia. J Innate Immun. 2016;8(5):517-528.

26. Lesbordes-Brion JC, et al. Targeted disruption of the hepcidin 1 gene results in severe hemochromatosis. Blood. 2006;108(4):1402-1405.

27. Nagababu E, Gulyani S, Earley CJ, Cutler RG, Mattson MP, Rifkind JM. Iron-deficiency anaemia enhances red blood cell oxidative stress. Free Radic Res. 2008;42(9):824-829.

28. Gosriwatana I, Loreal O, Lu S, Brissot P, Porter J, Hider RC. Quantification of non-transferrin-bound iron in the presence of unsaturated transferrin. Anal Biochem. 1999;273(2):212-220.

29. Breuer W, Ronson A, Slotki IN, Abramov A, Hershko C, Cabantchik ZI. The assessment of serum nontransferrin-bound iron in chelation therapy and iron supplementation. Blood. 2000;95(9):2975-2982.

30. Nassif X, Sansonetti PJ. Correlation of the virulence of Klebsiella pneumoniae K1 and K2 with the presence of a plasmid encoding aerobactin. Infect Immun. 1986;54(3):603-608

31. Pigeon C, et al. A new mouse liver-specific gene, encoding a protein homologous to human antimicrobial peptide hepcidin, is overexpressed during iron overload. J Biol Chem. 2001;276(11):7811-7819.

32. Wang L, Cherayil BJ. Ironing out the wrinkles in host defense: interactions between iron homeostasis and innate immunity. J Innate Immun. 2009;1(5):455-464

33. Georgopoulou U, Dimitriadis A, Foka P, Karamichali E, Mamalaki A. Hepcidin and the iron enigma in HCV infection. Virulence. 2014;5(4):465-476

34. Nahon P, Nuraldeen R, Rufat P, Sutton A, Trautwein C, Strnad P. In alcoholic cirrhosis, low-serum hepcidin levels associate with poor long-term survival. Liver Int. 2016;36(2):185-188.

35. Harrison-Findik DD, Klein E, Crist C, Evans J, Timchenko N, Gollan J. Iron-mediated regulation of liver hepcidin expression 
in rats and mice is abolished by alcohol. Hepatology. 2007;46(6):1979-1985.

36. Kessler SM, Barghash A, Laggai S, Helms V, Kiemer AK. Hepatic hepcidin expression is decreased in cirrhosis and HCC. J Hepatol. 2015;62(4):977-979.

37. Ramos E, et al. Minihepcidins prevent iron overload in a hepcidin-deficient mouse model of severe hemochromatosis. Blood. 2012;120(18):3829-3836.

38. Preza GC, et al. Minihepcidins are rationally designed small peptides that mimic hepcidin activity in mice and may be useful for the treatment of iron overload. J Clin Invest. 2011;121(12):4880-4888.

39. Krause A, et al. LEAP-1, a novel highly disulfide-bonded human peptide, exhibits antimicrobial activity. FEBS Lett. 2000;480(2-3):147-150.

40. Pigeon C, et al. A new mouse liver-specific gene, encoding a protein homologous to human antimicrobial peptide hepcidin, is overexpressed during iron overload. J Biol Chem. 2001;276(11):7811-7819.

41. Kim A, et al. A mouse model of anemia of inflammation: complex pathogenesis with partial dependence on hepcidin. Blood. 2014;123(8):1129-1136.

42. Kell DB, Pretorius E. Serum ferritin is an important inflammatory disease marker, as it is mainly a leakage product from damaged cells. Metallomics. 2014;6(4):748-773.

43. Nemeth E, et al. IL-6 mediates hypoferremia of inflammation by inducing the synthesis of the iron regulatory hormone hepcidin. J Clin Invest. 2004;113(9):1271-1276.

44. Podschun R, Ullmann U. Klebsiella spp. as nosocomial pathogens: epidemiology, taxonomy, typing methods, and pathogenicity factors. Clin Microbiol Rev. 1998;11(4):589-603.

45. Lawlor MS, O'connor C, Miller VL. Yersiniabactin is a virulence factor for Klebsiella pneumoniae during pulmonary infection. Infect Immun. 2007;75(3):1463-1472.

46. Lawlor MS, Hsu J, Rick PD, Miller VL. Identification of Klebsiella pneumoniae virulence determinants using an intranasal infection model. Mol Microbiol. 2005;58(4):1054-1073.

47. Bachman MA, Lenio S, Schmidt L, Oyler JE, Weiser JN. Interaction of lipocalin 2, transferrin, and siderophores determines the replicative niche of Klebsiella pneumoniae during pneumonia. MBio. 2012;3(6):6.

48. Bachman MA, Miller VL, Weiser JN. Mucosal lipocalin 2 has pro-inflammatory and iron-sequestering effects in response to bacterial enterobactin. PLoS Pathog. 2009;5(10):e1000622.

49. Bachman MA, et al. Klebsiella pneumoniae yersiniabactin promotes respiratory tract infection through evasion of lipocalin 2 . Infect Immun. 2011;79(8):3309-3316.

50. Drancourt M, Bollet C, Carta A, Rousselier P. Phylogenetic analyses of Klebsiella species delineate Klebsiella and Raoultella gen. nov., with description of Raoultella ornithinolytica comb. nov., Raoultella terrigena comb. nov. and Raoultella planticola comb. nov. Int J Syst Evol Microbiol. 2001;51(Pt 3):925-932.

51. Gustot T, Durand F, Lebrec D, Vincent JL, Moreau R. Severe sepsis in cirrhosis. Hepatology. 2009;50(6):2022-2033.

52. Jong GM, Hsiue TR, Chen CR, Chang HY, Chen CW. Rapidly fatal outcome of bacteremic Klebsiella pneumoniae pneumonia in alcoholics. Chest. 1995;107(1):214-217.

53. Pak M, Lopez MA, Gabayan V, Ganz T, Rivera S. Suppression of hepcidin during anemia requires erythropoietic activity. Blood. 2006;108(12):3730-3735.

54. Duong-Nu TM, et al. All Three TonB Systems Are Required for Vibrio vulnificus CMCP6 Tissue Invasiveness by Controlling Flagellum Expression. Infect Immun. 2015;84(1):254-265.

55. Elin RJ, Wolff SM. The role of iron in nonspecific resistance to infection induced by endotoxin. J Immunol. 1974;112(2):737-745.

56. Mehrad B, et al. Transient lung-specific expression of the chemokine KC improves outcome in invasive aspergillosis. Am J Respir Crit Care Med. 2002;166(9):1263-1268.

57. Mehrad B, et al. The lupus-susceptibility locus, Sle3, mediates enhanced resistance to bacterial infections. J Immunol. 2006;176(5):3233-3239

58. Guo Z, et al. Cutting edge: membrane lymphotoxin regulates CD8(+) T cell-mediated intestinal allograft rejection. J Immunol. 2001;167(9):4796-4800.

59. Grundy MA, Gorman N, Sinclair PR, Chorney MJ, Gerhard GS. High-throughput non-heme iron assay for animal tissues. J Biochem Biophys Methods. 2004;59(2):195-200.

60. Rebouche CJ, Wilcox CL, Widness JA. Microanalysis of non-heme iron in animal tissues. J Biochem Biophys Methods. 2004;58(3):239-251.

61. Oremland M, Michels KR, Bettina AM, Lawrence C, Mehrad B, Laubenbacher R. A computational model of invasive aspergillosis in the lung and the role of iron. BMC Syst Biol. 2016;10:34.

62. Esposito BP, Breuer W, Sirankapracha P, Pootrakul P, Hershko C, Cabantchik ZI. Labile plasma iron in iron overload: redox activity and susceptibility to chelation. Blood. 2003;102(7):2670-2677.

63. Barletta KE, Cagnina RE, Wallace KL, Ramos SI, Mehrad B, Linden J. Leukocyte compartments in the mouse lung: distinguishing between marginated, interstitial, and alveolar cells in response to injury. J Immunol Methods. 2012;375(1-2):100-110

64. Misharin AV, Morales-Nebreda L, Mutlu GM, Budinger GR, Perlman H. Flow cytometric analysis of macrophages and dendritic cell subsets in the mouse lung. Am J Respir Cell Mol Biol. 2013;49(4):503-510 